\title{
TGA studies of metoclopramide complexes of cobalt(II) in the solid state
}

\author{
P.R. Chetana ${ }^{\mathrm{a}, *}$, X. Siddaramaiah ${ }^{\mathrm{b}}$, P.G. Ramappa ${ }^{\mathrm{c}}$ \\ a Department of Post Graduate Studies in Chemistry, University of Bangalore, Central College Campus, Bangalore 560001, India \\ ${ }^{\mathrm{b}}$ Department of Polymer Science and Technology, Sri Jayachamarajendra College of Engineering, Mysore 570 006, India \\ ${ }^{c}$ Department of Post Graduate Studies in Chemistry, University of Mysore, Manasagangothri, Mysore 570 006, India
}

Received 21 April 2003; received in revised form 2 October 2003; accepted 20 October 2003

\begin{abstract}
A new series of cobalt(II) complexes with metoclopramide (MCP) ligand have been prepared. The prepared Co(II)-MCP complexes were characterized for various analytical techniques. Conductivity and elemental analysis of complexes have been measured. The thermal stability and degradation kinetics have been measured using thermogravimetric analyser. Kinetic parameter was obtained for each stage of thermal degradation for Co(II)-MCP complexes using Horowitz-Metzger, Coats-Redfern and Broido's methods. The activation energy $\left(E_{\mathrm{a}}\right)$ of the complexes for the thermal degradation process lie in the range 31-168, 23-161 and 33-170 kJ mol ${ }^{-1}$ for Horowitz-Metzger, Coats-Redfern and Broido's methods, respectively.
\end{abstract}

(C) 2003 Elsevier B.V. All rights reserved.

Keywords: Cobalt(II); Metoclopramide; Complexes; TG; Thermal degradation kinetics

\section{Introduction}

Benzamide-4-amino-5-chloro- $N$-[2-(diethyl amine)ethyl]2-methoxy monochloride, metoclopramide (MCP), was prepared by Murakami et al. [1]. MCP is a dopamine antagonist clinically used as antiemetics. This drug has therapeutic potential and its mechanism of action at receptor and molecular level was described [2]. MCP is used as a reagent for the extraction-spectrophotometric determination of ion association complex rhenium(IV)-thiocyanate [3,4]. The presence of amide nitrogen and tertiary nitrogen in the structure of MCP ligand enables it to form complexes with transition metals. Metal complexes are extensively used as catalysts, optical modifier, conducting filler, antiradator filler, etc. To select the suitable material for given applications material technologists requires knowledge about their properties. The knowledge of their physico-chemical and thermal properties is useful to exploit these complexes for newer applications. Survey of the literature reveals that the synthesis and characterization of $\mathrm{Co}(\mathrm{II})-\mathrm{MCP}$ complexes have not been studied.

\footnotetext{
* Corresponding author. Fax: +91-80-2386988.

E-mail address: prchetana@hotmial.com (P.R. Chetana).
}

The present research article deals with the synthesis, physical properties and thermal degradation kinetics of new series of Co(II) complexes with MCP ligand. The thermal degradation kinetic parameters such as energy of activation $\left(E_{\mathrm{a}}\right)$ and frequency factor $(\ln A)$; and thermodynamic parameters like change in entropy $(\Delta S)$, change in enthalpy $(\Delta H)$ and change in free energy $(\Delta G)$ for each step have been evaluated. The kinetic parameters have been calculated using three relations like Horowitz-Metzger [5], Coats-Redfern [6] and Broido's [7] methods and compared.

\section{Experimental}

\subsection{Materials}

Cobalt salts and solvents used were of AR grade. The ligand MCP was obtained as a gift sample from Astra IDL Ltd., Bangalore, India. It is used as it is without further purification.

\subsection{Preparation of complexes}

An ethanolic solution of $50 \mathrm{ml}$ of $5 \mathrm{mM}$ MCP was slowly added to $50 \mathrm{ml}$ of $5 \mathrm{mM}$ ethanolic $\mathrm{Co}$ (II) salt with continu- 
ous stirring. The mixture was then refluxed in water bath at $65-80^{\circ} \mathrm{C}$ for $2 \mathrm{~h}$. The blue coloured precipitate was obtained in each case. The solid complexes formed were cooled, filtered, and washed successively with ethanol and then with ether. The Co(II) complexes were finally dried in a vacuum desiccator over anhydrous silica gel.

\subsection{Physical measurements}

Magnetic moments were measured by employing Gouy balance. The conductivity measurements were carried out using Equiptronics digital conductivity meter model EQ-DCM-P type at room temperature for $1 \times 10^{-3} \mathrm{M}$ solutions. The hydrogen and carbon content of the complexes have been measured by microelemental procedures. The metal content was determined by complexometric method [8].

\subsection{Treatment of TGA data}

The thermal degradation parameters of Co(II)-MCP complexes were evaluated using DuPont 9900 TA with 951 TGA module. About $6-8 \mathrm{mg}$ of pure sample was subjected to dynamic TGA scans at a heating rate of $10^{\circ} \mathrm{C} \mathrm{min}^{-1}$ in the temperature range of ambient to $900^{\circ} \mathrm{C}$ in $\mathrm{N}_{2}$ atmosphere.

The TG curves were analysed as percentage weight loss as a function of temperature. The three decomposition steps were identified using a derivative of the TG curves. The kinetic and thermodynamic parameters of thermal degradation process have been calculated using three models.

The Horowitz-Metzger [5] relation used to evaluate the degradation kinetics is

$\ln \left[\frac{\ln \left(W_{0}-W_{\mathrm{t}}^{\mathrm{f}}\right)}{W-W_{\mathrm{t}}^{\mathrm{f}}}\right]=\frac{E_{\mathrm{a}} \theta}{R T_{\mathrm{s}}^{2}}$

where $W_{0}$ is the initial weight of the sample, $W_{\mathrm{t}}^{\mathrm{f}}$ the final weight of the sample, $W$ the weight remaining at a given temperature, $T, E_{\mathrm{a}}$ the activation energy, $\theta=T-T_{\mathrm{s}}, T_{\mathrm{s}}$ the DTG peak temperature, $T$ the temperature corresponding to weight loss, $W$.

Coats-Redfern [6] relation is as follows:

$\log \left\{-\log \left[\frac{1-\alpha}{T^{2}}\right]=\log \left\{\left(\frac{A R}{a E_{\mathrm{a}}}\right)-\left(\frac{E_{\mathrm{a}}}{2.3 R T}\right)\right\}\right\}$

where $\alpha$ is the fraction of sample decomposed at time $T, T$ the derivative peak temperature, $A$ the frequency factor, $a$ the heating rate, $E_{\mathrm{a}}$ the activation energy, $R$ the gas constant.

A plot of $\log \left\{-\log (1-\alpha) / T^{2}\right\}$ versus $1 / T$ gives the slope for evaluation of the activation energy most appropriately.

Mathematical expression of Broido's [7] method is as follows:

$\ln \left\{\ln \left(\frac{1}{y}\right)\right\}=\left\{\left(-\frac{E_{\mathrm{a}}}{R}\right)\left(\frac{1}{T}\right)\right\}$

where $y$ is the fraction of number of initial molecules not yet decomposed, $T$ the peak temperature of derivative curve of TG, $R$ the gas constant, $E_{\mathrm{a}}$ the activation energy can be calculated from the plot of $\ln \{\ln (1 / y)\}$ versus $1 / T$.

\section{Results and discussion}

\subsection{Physical analysis}

All the synthesized divalent cobalt complexes with MCP were blue in colour, and nonhygroscopic towards air and moisture. The elemental analysis results obtained by microanalytical measurement and metal content determination data indicate 1:1 metal to ligand stoichiometry. The molar conductance values of these complexes $\left(1.0 \times 10^{-3} \mathrm{M}\right.$ in DMF) lie in the range $27.76-44.58 \mathrm{mho} \mathrm{cm}^{-1} \mathrm{~mol}^{-1}$, which reveals that these complexes are nonelectrolytes.

\subsection{Thermogravimetric analysis}

TGA and its derivative thermograms for MCP and cobalt complexes are shown in Figs. 1-2, respectively. From the thermograms it can be observed that two-step thermal degradation processes for MCP, whereas its cobalt complexes shows three-step thermal degradation behaviour. In order to determine the thermal stability trend the temperature characteristics such as $T_{0}$ (temperature at the onset of decomposition), $T_{10}$ (temperature at $10 \%$ weight loss), $T_{20}$ (temperature at $20 \%$ weight loss), $T_{50}$ (temperature at $50 \%$ weight loss), $T_{\max }$ (temperature at maximum rate of decomposition) and integral procedure for decomposition temperature (IPDT) values of the degradation process were calculated (Table 1).

$T_{0}, T_{10}, T_{20}$ and $T_{\max }$ are the main criteria of the thermal stability of complexes. The higher the value of $T_{0}, T_{10}, T_{20}$ and $T_{\max }$, higher will be the heat stability of complexes [9]. To obtain the quantitative picture of the relative stability, IPDT values can be regarded of significant importance, since they represent the overall nature of the thermogravimetric curves.

Chemical structure and nature of inorganic ligands will affect the thermal stability and degradation pattern of Co(II)-MCP complexes. From Table 1 it was noticed that the thermal stability of all $\mathrm{Co}$ (II)-MCP complexes are lesser than $128^{\circ} \mathrm{C}$ except $\left[\mathrm{Co}(\mathrm{MCP})(\mathrm{SCN})_{2}\right]$ complex. This is due to the presence of water ligand in all other complexes. From Table 1, it was noticed that high IPDT value of $586^{\circ} \mathrm{C}$ for $\left[\mathrm{Co}(\mathrm{MCP})(\mathrm{SCN})_{2}\right]$ compared to other inorganic ligands. The greater thermal stability of $\left[\mathrm{Co}(\mathrm{MCP})(\mathrm{SCN})_{2}\right]$ may be ascribed to greater heat resistance character of -SCN linkage compared with those of other inner sphere inorganic ligands [10,11]. The lowest thermal stability of $\left[\mathrm{Co}(\mathrm{MCP}) \mathrm{Br}_{2}\right]$ complex may be due to low electronegative nature of bromide ion. The trend of thermal degradation stability for the complexes are given in Tables 1 and 2 . 


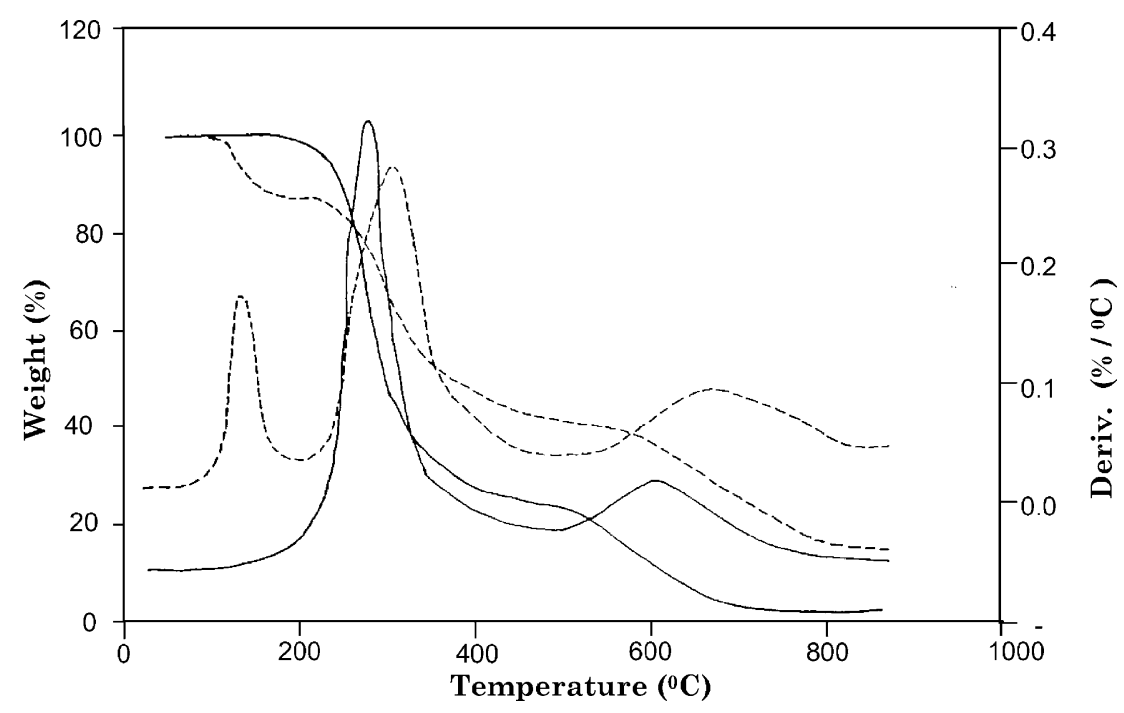

Fig. 1. TGA and derivative thermograms of $\mathrm{MCP}(-)$ and $\left[\mathrm{Co}(\mathrm{MCP})\left(\mathrm{H}_{2} \mathrm{O}\right)_{2} \mathrm{SO}_{4}\right]$ (---).

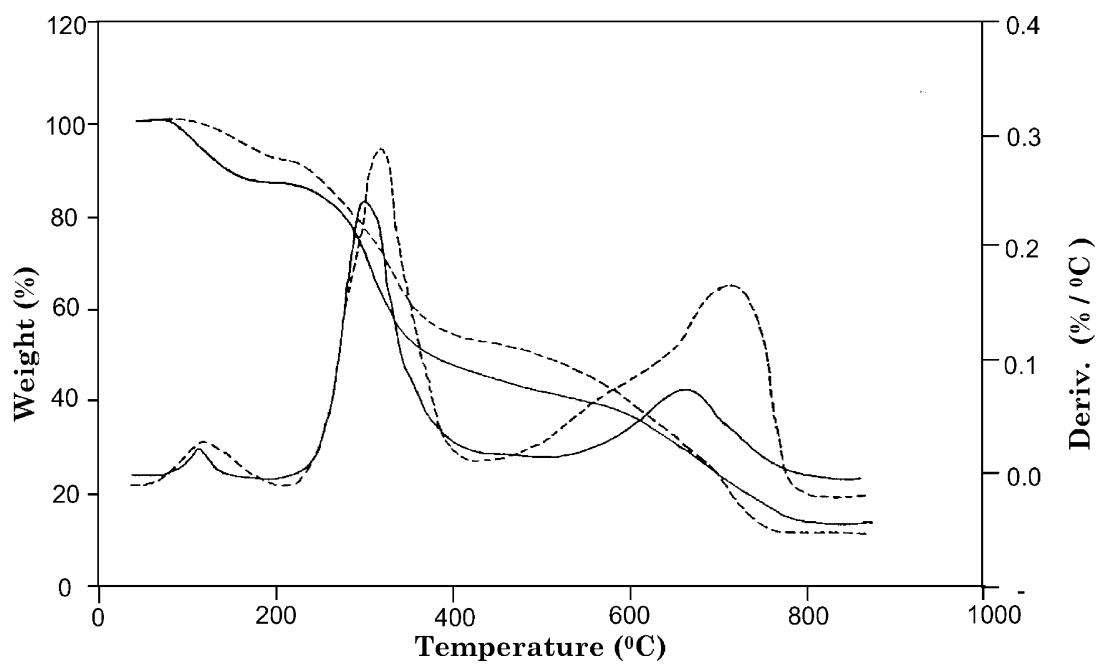

Fig. 2. TGA and derivative thermograms of $\left[\mathrm{Co}(\mathrm{MCP})\left(\mathrm{H}_{2} \mathrm{O}\right)_{2} \mathrm{Cl}_{2}\right](-)$ and $\left[\mathrm{Co}(\mathrm{MCP})\left(\mathrm{H}_{2} \mathrm{O}\right)_{2} \mathrm{Br}_{2}\right](---)$.

\subsubsection{First stage of thermal decomposition}

The first step of thermal decomposition of Co(II) complexes starts at $105^{\circ} \mathrm{C}$ and is marked with a regular loss in mass up to $275^{\circ} \mathrm{C}$. The initial mass loss for the complexes varies from 6.2 to $7.8 \%$, obtained in pyrolysis and agrees well with the theoretically expected loss of $6.5-7.3 \%$. This is mainly due to the elimination of two-molecule of coordinated water $[11,12]$.

Table 1

Characteristic transition temperature and IPDT values of Co-MCP complexes obtained by TGA scans

\begin{tabular}{lllllll}
\hline Complexes & \multicolumn{3}{l}{ Temperature at different weight loss $+2{ }^{\circ} \mathrm{C}$} & & \\
\cline { 2 - 7 } & $T_{0}$ & $T_{10}$ & $T_{20}$ & $T_{50}$ & $T_{\max }$ & IPDT \\
\hline $\mathrm{MCP}$ & 235 & 248 & 262 & 295 & 656 & 497 \\
{$\left[\mathrm{Co}(\mathrm{MCP})\left(\mathrm{H}_{2} \mathrm{O}\right)_{2}\left(\mathrm{ClO}_{4}\right)_{2}\right]$} & 225 & 267 & 271 & 283 & 564 & 414 \\
{$\left[\mathrm{Co}(\mathrm{MCP})\left(\mathrm{H}_{2} \mathrm{O}\right)_{2} \mathrm{Br}_{2}\right]$} & 110 & 193 & 290 & 509 & 761 & 554 \\
{$\left[\mathrm{Co}(\mathrm{MCP})\left(\mathrm{H}_{2} \mathrm{O}\right)_{2} \mathrm{Cl}_{2}\right]$} & 108 & 148 & 270 & 389 & 795 & 500 \\
{$\left[\mathrm{Co}(\mathrm{MCP})\left(\mathrm{H}_{2} \mathrm{O}\right)_{2} \mathrm{SO}_{4}\right]$} & 121 & 145 & 267 & 374 & 890 & 523 \\
{$\left[\mathrm{Co}(\mathrm{MCP})\left(\mathrm{NCS}_{2}\right]\right.$} & 208 & 280 & 292 & 446 & 656 & 486 \\
{$\left[\mathrm{Co}(\mathrm{MCP})\left(\mathrm{H}_{2} \mathrm{O}\right)_{2}\left(\mathrm{NO}_{3}\right)_{2}\right]$} & 106 & 238 & 296 & 451 & 675 & 490 \\
{$\left[\mathrm{Co}(\mathrm{MCP})\left(\mathrm{H}_{2} \mathrm{O}\right)_{2}\left(\mathrm{CH}_{3} \mathrm{COO}\right)_{2}\right]$} & 128 & 193 & 277 & 406 & 634 & 538 \\
\hline
\end{tabular}


Table 2

Stepwise thermal degradation data obtained from TGA curves for Co(II)-MCP complexes

\begin{tabular}{|c|c|c|c|c|c|c|c|c|c|c|}
\hline \multirow[t]{2}{*}{ Complexes } & \multirow[t]{2}{*}{ Process } & \multirow[t]{2}{*}{ Temperature range $\left({ }^{\circ} \mathrm{C}\right)$} & \multirow[t]{2}{*}{$T_{\max }\left({ }^{\circ} \mathrm{C}\right)$} & \multirow[t]{2}{*}{ Products } & \multicolumn{2}{|c|}{ Weight loss (\%) } & \multirow[t]{2}{*}{ No. of moles } & \multicolumn{2}{|c|}{ Residue (\%) } & \multirow[t]{2}{*}{ Nature } \\
\hline & & & & & Calculated & Experimental & & Calculated & Experimental & \\
\hline \multirow[t]{2}{*}{ MCP } & I & $235-440$ & 276 & $\mathrm{~L}$ & - & 75.4 & 0.8 & - & 0.2 & \\
\hline & II & $459-705$ & 594 & $\mathrm{~L}$ & - & 24.4 & 0.2 & & & \\
\hline \multirow[t]{2}{*}[\mathrm{Co}(\mathrm{MCP})(\mathrm{H}_{2}\mathrm{O})_{2}(\mathrm{ClO}_{4})_{2}]{} & I & $225-305$ & 278 & $\mathrm{H}_{2} \mathrm{O}+\mathrm{ClO}_{4}+\mathrm{L}$ & 78.8 & 69.7 & $2.0+2.0+0.8$ & 12.1 & 3.8 & $\mathrm{CoO}$ \\
\hline & II & $331-590$ & 499 & $\mathrm{~L}$ & 12.6 & 26.8 & & & & \\
\hline \multirow[t]{3}{*}[\mathrm{Co}(\mathrm{MCP})(\mathrm{H}_{2}\mathrm{O})_{2}\mathrm{Br}_{2}]{} & I & $110-199$ & 129 & $\mathrm{H}_{2} \mathrm{O}$ & 6.5 & 6.1 & 2.0 & 13.5 & 11.7 & $\mathrm{CoO}$ \\
\hline & II & $215-410$ & 322 & $\mathrm{~L}$ & 40.5 & 40.2 & 0.8 & & & \\
\hline & III & $414-770$ & 716 & $\mathrm{~L}+\mathrm{Br}$ & 41.8 & 42.0 & $0.2+2.0$ & & & \\
\hline \multirow{3}{*}[\mathrm{Co}(\mathrm{MCP})(\mathrm{H}_{2}\mathrm{O})_{2}\mathrm{Cl}_{2}]{} & I & $108-176$ & 116 & $\mathrm{H}_{2} \mathrm{O}$ & 7.7 & 7.8 & 2.0 & 16.1 & 16.0 & $\mathrm{CoO}$ \\
\hline & II & $215-510$ & 303 & $\mathrm{~L}$ & 48.3 & 46.6 & 0.8 & & & \\
\hline & III & $416-820$ & 720 & $\mathrm{~L}+\mathrm{Cl}$ & 30.7 & 29.6 & $0.2+2.0$ & & & \\
\hline \multirow[t]{3}{*}[\mathrm{Co}(\mathrm{MCP})(\mathrm{H}_{2}\mathrm{O})_{2}\mathrm{SO}_{4}]{} & I & $121-201$ & 135 & $\mathrm{H}_{2} \mathrm{O}$ & 7.3 & 7.4 & 2.0 & 15.4 & 15.1 & $\mathrm{CoO}$ \\
\hline & II & $201-489$ & 302 & $\mathrm{~L}$ & 45.8 & 45.6 & & & & \\
\hline & III & $489-844$ & 661 & $\mathrm{~L}+\mathrm{SO}_{4}$ & 35.0 & 32.6 & $0.2+1.0$ & & & \\
\hline \multirow[t]{3}{*}[\mathrm{Co}(\mathrm{MCP})(\mathrm{NCS})_{2}]{} & I & $208-375$ & 236 & $\mathrm{~L}$ & 47.7 & 46.8 & 0.8 & 15.8 & 15.8 & $\mathrm{CoO}$ \\
\hline & II & $375-410$ & 345 & $\mathrm{~L}+\mathrm{CN}$ & 26.1 & 25.0 & $0.2+2.0$ & & & \\
\hline & III & $525-805$ & 738 & $\mathrm{~S}$ & 13.5 & 12.3 & 2.0 & & & \\
\hline \multirow[t]{3}{*}[\mathrm{Co}(\mathrm{MCP})(\mathrm{H}_{2}\mathrm{O})_{2}(\mathrm{NO}_{3})_{2}]{} & I & $106-206$ & 114 & $\mathrm{H}_{2} \mathrm{O}$ & 6.9 & 6.7 & 2.0 & 14.3 & 14.4 & $\mathrm{CoO}$ \\
\hline & II & $213-435$ & 335 & $\mathrm{~L}$ & 42.6 & 41.5 & 0.8 & & & \\
\hline & III & $490-630$ & 728 & $\mathrm{~L}+\mathrm{NO}_{3}$ & 38.5 & 37.8 & $0.2+2.0$ & & & \\
\hline \multirow{3}{*}[\mathrm{Co}(\mathrm{MCP})(\mathrm{H}_{2}\mathrm{O})_{2}(\mathrm{CH}_{3}\mathrm{COO})_{2}]{} & I & $128-216$ & 162 & $\mathrm{H}_{2} \mathrm{O}$ & 7.0 & 7.1 & 2.0 & 14.6 & 14.2 & $\mathrm{CoO}$ \\
\hline & II & $218-430$ & 327 & $\mathrm{~L}$ & 43.8 & 43.6 & 0.8 & & & \\
\hline & III & $505-742$ & 634 & $\mathrm{~L}+\mathrm{CH}_{3} \mathrm{COO}$ & 37.1 & 35.1 & $0.2+2.0$ & & & \\
\hline
\end{tabular}


A realistic explanation for the anion effect can be provided by defect diffusion model [13]. When a reaction takes place in a solid, the formation of transition state results in a point defect in the lattice. These defects are analogous to the substituted ion Schotty and Frenkel defects [14]. When a volatile ligand like water is lost from a complex, a situation analogous to the Frenkel defect is formed. This introduces distortion in the structure of the crystal leading to transition state. The fraction of free space in crystal increases as the difference between the size of the cation and anion increases $[15,16]$. The activation energies in Table 3 of the reported Co(II)-MCP complexes for deaquation reaction decreases in the order perchlorate $>$ sulphate $>$ acetate $>$ bromide $>$ chloride $>$ nitrate. The fraction of free space is much lower when cation and anions are of equal size. Therefore in perchlorate compounds deaquation reactions take place with difficulty [17].

\subsubsection{Second stage of thermal decomposition}

The major weight loss of about 40-48\% occurred in second step degradation process in the temperature range $200-510^{\circ} \mathrm{C}$. The weight loss in this step is due to partial decomposition of organic ligand. The degradation pattern is on par with that of the MCP ligand. The activation energy values for this step are in the range $38.2-76.4 \mathrm{~kJ} \mathrm{~mol}^{-1}$ in Horowitz-Metzger method that are comparable with Coats-Redfern and Broido's methods.

\subsubsection{Third stage of thermal decomposition}

The mass loss in the temperature range $414-844{ }^{\circ} \mathrm{C}$ corresponds to third step degradation process of complexes. The weight loss in this step is about $29.5-42.0 \%$. The weight loss in this step is due to the elimination of inorganic ligand and $25 \%$ of MCP ligand. The residue of pyrolysis of complexes corresponds to cobalt oxide [18], which was chemically confirmed. In all the three degradations of all complexes there is correlation between the calculated and the experimental weight losses.

Though the Co(II) thiocyanate complex show three stage degradation, the pattern is different from that of other complexes [19,20]. The major portion of MCP ligand is eliminated in the first stage degradation, the elimination of remaining portion of MCP ligand and $(\mathrm{CN})_{2}$ occurred in second stage degradation. Sulphur is eliminated in the third stage of the degradation process.

Co(II)-perchlorate complex shows only two-step decompositions. It is stable up to $277^{\circ} \mathrm{C}$ and rapidly decomposes at this step; weight loss is about $69.7 \%$. The first stage corresponds to elimination of a part of the MCP ligand, water molecules and perchlorate ions. In the second stage the remaining portion of the MCP ligand decomposes. The sudden decomposition of this complex may be due to the explosive nature of perchlorate ion present in it. This complex shows a different type of decomposition from those of other complexes. This suggests that the ligand water molecule be

Table 3

Activation energy and correlation co-efficient values for the thermal degradation of Co-MCP complexes

\begin{tabular}{|c|c|c|c|c|c|c|c|}
\hline \multirow[t]{2}{*}{ Compounds } & \multirow[t]{2}{*}{ Process } & \multicolumn{3}{|c|}{$E_{\mathrm{a}} \pm 2 \%\left(\mathrm{~kJ} \mathrm{~mol}^{-1}\right)$} & \multicolumn{3}{|c|}{ Correlation co-efficient, $\left|r^{2}\right|$} \\
\hline & & H-M & $\mathrm{C}-\mathrm{R}$ & Broido's & $\mathrm{H}-\mathrm{M}$ & C-R & Broido's \\
\hline \multirow[t]{2}{*}{ MCP } & I & 88.4 & 79.3 & 88.5 & 0.975 & 0.976 & 0.981 \\
\hline & II & 31.2 & 19.1 & 33.5 & 0.988 & 0.957 & 0.986 \\
\hline \multirow[t]{2}{*}[\mathrm{Co}(\mathrm{MCP})(\mathrm{H}_{2}\mathrm{O})_{2}(\mathrm{ClO}_{4})_{2}]{} & I & 168.7 & 161.4 & 170.6 & 0.732 & 0.710 & 0.733 \\
\hline & II & 38.2 & 25.4 & 38.3 & 0.988 & 0.976 & 0.989 \\
\hline \multirow[t]{3}{*}[\mathrm{Co}(\mathrm{MCP})(\mathrm{H}_{2}\mathrm{O})_{2}\mathrm{Br}_{2}]{} & I & 45.5 & 44.2 & 45.4 & 0.963 & 0.955 & 0.956 \\
\hline & II & 44.2 & 34.2 & 44.1 & 0.998 & 0.997 & 0.998 \\
\hline & III & 62.6 & 46.0 & 62.4 & 0.995 & 0.989 & 0.994 \\
\hline \multirow[t]{3}{*}[\mathrm{Co}(\mathrm{MCP})(\mathrm{H}_{2}\mathrm{O})_{2}\mathrm{Cl}_{2}]{} & I & 37.4 & 43.6 & 40.2 & 0.882 & 0.862 & 0.901 \\
\hline & II & 54.9 & 45.2 & 54.8 & 0.954 & 0.988 & 0.993 \\
\hline & III & 33.6 & 23.8 & 29.3 & 0.987 & 0.944 & 0.987 \\
\hline \multirow[t]{3}{*}[\mathrm{Co}(\mathrm{MCP})(\mathrm{H}_{2}\mathrm{O})_{2}\mathrm{SO}_{4}]{} & I & 51.9 & 55.2 & 51.9 & 0.961 & 0.960 & 0.970 \\
\hline & II & 44.2 & 34.8 & 44.4 & 0.977 & 0.973 & 0.984 \\
\hline & III & 23.2 & 17.8 & 23.3 & 0.987 & 0.903 & 0.989 \\
\hline \multirow[t]{3}{*}[\mathrm{Co}(\mathrm{MCP})(\mathrm{NCS})_{2}]{} & I & 72.6 & 68.7 & 74.3 & 0.987 & 0.903 & 0.989 \\
\hline & II & 76.2 & 73.6 & 65.5 & 0.995 & 0.906 & 0.923 \\
\hline & III & 54.0 & 48.0 & 52.8 & 0.981 & 0.968 & 0.915 \\
\hline \multirow[t]{3}{*}[\mathrm{Co}(\mathrm{MCP})(\mathrm{H}_{2}\mathrm{O})_{2}(\mathrm{NO}_{3})_{2}]{} & I & 35.6 & 41.0 & 36.5 & 0.984 & 0.896 & 0.982 \\
\hline & II & 48.7 & 37.5 & 59.4 & 0.915 & 0.906 & 0.894 \\
\hline & III & 51.5 & 49.4 & 51.9 & 0.962 & 0.987 & 0.922 \\
\hline \multirow[t]{3}{*}[\mathrm{Co}(\mathrm{MCP})(\mathrm{H}_{2}\mathrm{O})_{2}(\mathrm{CH}_{3}\mathrm{COO})_{2}]{} & I & 51.5 & 52.3 & 50.35 & 0.957 & 0.891 & 0.980 \\
\hline & II & 76.4 & 74.0 & 76.9 & 0.908 & 0.901 & 0.996 \\
\hline & III & 69.3 & 64.5 & 68.5 & 0.889 & 0.893 & 0.959 \\
\hline
\end{tabular}


retained even at a high temperature, since the bulky perchlorate ion cannot easily migrate towards the inner sphere [21]. This fact seems to account for the mechanism by which the deaquation reaction is initiated by the migration of the anion as explained by defect diffusion model.

\subsubsection{Degradation mechanism}

The broad outline for thermal decomposition mechanism of $\mathrm{Co}(\mathrm{II})-\mathrm{MCP}$ complexes is as follows:

- First step degradation

$$
\left[\mathrm{Co}(\mathrm{MCP})\left(\mathrm{H}_{2} \mathrm{O}\right)_{2} \mathrm{X}_{2}\right] \stackrel{105-2160^{\circ} \mathrm{C}}{\longrightarrow}\left[\mathrm{Co}(\mathrm{MCP}) \mathrm{X}_{2}\right]
$$

- Second step degradation

$$
\left[\mathrm{Co}(\mathrm{MCP}) \mathrm{X}_{2}\right] \stackrel{201-4100^{\circ} \mathrm{C}}{\longrightarrow}\left[\mathrm{Co}(\mathrm{MCP})_{0.25} \mathrm{X}_{2}\right]
$$

- Third step degradation

$$
\left[\mathrm{Co}(\mathrm{MCP})_{0.25} \mathrm{X}_{2}\right] \stackrel{414-844^{\circ} \mathrm{C}}{\longrightarrow} \mathrm{CoO}
$$

where $\mathrm{X}=\mathrm{Cl}^{-}, \mathrm{Br}^{-},(1 / 2) \mathrm{SO}_{4}{ }^{2-} \cdot \mathrm{NO}_{3}{ }^{-}$or $\mathrm{CH}_{3} \mathrm{COO}^{-}$.

The decomposition pattern in the $\mathrm{Co}(\mathrm{SCN})-\mathrm{MCP}$ complex is as follows:

- First step degradation

$$
\left[\mathrm{Co}(\mathrm{MCP})(\mathrm{SCN})_{2}\right] \stackrel{208-3750^{\circ} \mathrm{C}}{\longrightarrow}\left[\mathrm{Co}(\mathrm{MCP})_{0.25}(\mathrm{SCN})_{2}\right]
$$

- Second step degradation

$$
\left[\mathrm{Co}(\mathrm{MCP})_{0.25}(\mathrm{SCN})_{2}\right] \stackrel{375-4100^{\circ} \mathrm{C}}{\longrightarrow}\left[\mathrm{CoS}_{2}\right]
$$

- Third step degradation

$$
\left[\mathrm{CoS}_{2}\right] \stackrel{525-805^{\circ} \mathrm{C}}{\longrightarrow} \mathrm{CoO}
$$

The decomposition of Co(II)-perchlorate complex may be formulated as:

- First step degradation

$$
\left[\mathrm{Co}(\mathrm{MCP})\left(\mathrm{H}_{2} \mathrm{O}\right)_{2}\left(\mathrm{ClO}_{4}\right)_{2}\right] \stackrel{160-305^{\circ} \mathrm{C}}{\longrightarrow}\left[\mathrm{Co}(\mathrm{MCP})_{0.25}\right]
$$

- Second step degradation

$$
\left[\mathrm{Co}(\mathrm{MCP})_{0.25} \stackrel{331-590^{\circ} \mathrm{C}}{\longrightarrow} \mathrm{CoO}\right.
$$

\subsection{Kinetics and thermodynamics applied to pyrolysis}

The three decomposition steps of the complexes were subjected to nonisothermal kinetic and thermodynamic studies and the parameters such as apparent activation en-

\begin{tabular}{|c|c|c|c|c|c|c|c|}
\hline \multirow[t]{2}{*}{ Compounds } & \multirow[t]{2}{*}{ Process } & \multicolumn{3}{|c|}{$\ln A \pm 2\left(\min ^{-1}\right)$} & \multicolumn{3}{|c|}{$K_{\mathrm{r}}\left(\min ^{-1}\right)$} \\
\hline & & $\mathrm{H}-\mathrm{M}$ & $\mathrm{C}-\mathrm{R}$ & Broido's & $\mathrm{H}-\mathrm{M}$ & C-R & Broido's \\
\hline \multirow[t]{2}{*}{ MCP } & I & 19.0 & 16.0 & 19.0 & 0.702 & 0.246 & 0.702 \\
\hline & II & 2.0 & 0.7 & 2.3 & 0.100 & 0.135 & 0.107 \\
\hline \multirow[t]{2}{*}[\mathrm{Co}(\mathrm{MCP})(\mathrm{H}_{2}\mathrm{O})_{2}(\mathrm{ClO}_{4})_{2}]{} & I & 37.2 & 35.0 & 37.6 & 1.340 & 0.617 & 1.355 \\
\hline & II & 4.1 & 2.5 & 4.1 & 0.154 & 0.223 & 0.154 \\
\hline \multirow[t]{3}{*}[\mathrm{Co}(\mathrm{MCP})(\mathrm{H}_{2}\mathrm{O})_{2}\mathrm{Br}_{2}]{} & I & 13.2 & 7.2 & 13.2 & 0.677 & 0.002 & 0.676 \\
\hline & II & 7.8 & 4.4 & 7.7 & 0.300 & 0.082 & 0.299 \\
\hline & III & 5.8 & 3.9 & 5.7 & 0.154 & 0.182 & 0.154 \\
\hline \multirow[t]{3}{*}[\mathrm{Co}(\mathrm{MCP})(\mathrm{H}_{2}\mathrm{O})_{2}\mathrm{Cl}_{2}]{} & I & 11.0 & 6.7 & 12.0 & 0.594 & 0.025 & 0.639 \\
\hline & II & 10.6 & 7.2 & 10.5 & 0.398 & 0.104 & 0.397 \\
\hline & III & 1.6 & 1.2 & 0.9 & 0.082 & 0.199 & 0.072 \\
\hline \multirow[t]{3}{*}[\mathrm{Co}(\mathrm{MCP})(\mathrm{H}_{2}\mathrm{O})_{2}\mathrm{SO}_{4}]{} & I & 15.0 & 10.0 & 15.0 & 0.748 & 0.038 & 0.749 \\
\hline & II & 8.1 & 5.0 & 8.1 & 0.320 & 0.101 & 0.321 \\
\hline & III & 0.2 & 0.2 & 0.3 & 0.064 & 0.124 & 0.064 \\
\hline \multirow[t]{3}{*}[\mathrm{Co}(\mathrm{MCP})(\mathrm{NCS})_{2}]{} & I & 16.7 & 10.8 & 17.2 & 0.672 & 0.004 & 0.688 \\
\hline & II & 14.1 & 7.9 & 11.9 & 0.481 & 0.002 & 0.413 \\
\hline & III & 4.4 & 3.7 & 4.2 & 0.127 & 0.130 & 0.124 \\
\hline \multirow[t]{3}{*}[\mathrm{Co}(\mathrm{MCP})(\mathrm{H}_{2}\mathrm{O})_{2}(\mathrm{NO}_{3})_{2}]{} & I & 13.9 & 10.6 & 14.2 & 0.733 & 0.119 & 0.748 \\
\hline & II & 8.5 & 6.2 & 10.8 & 0.317 & 0.291 & 0.386 \\
\hline & III & 4.1 & 3.5 & 4.2 & 0.124 & 0.084 & 0.125 \\
\hline \multirow[t]{3}{*}[\mathrm{Co}(\mathrm{MCP})(\mathrm{H}_{2}\mathrm{O})_{2}(\mathrm{CH}_{3}\mathrm{COO})_{2}]{} & I & 13.8 & 10.1 & 13.4 & 0.653 & 0.013 & 0.638 \\
\hline & II & 14.7 & 9.7 & 14.8 & 0.511 & 0.006 & 0.515 \\
\hline & III & 7.6 & 5.5 & 7.5 & 0.203 & 0.047 & 0.200 \\
\hline
\end{tabular}
ergy $\left(E_{\mathrm{a}}\right)$, pre-exponential factor $(A)$, rate constant $\left(K_{\mathrm{r}}\right)$, entropy of activation $(\Delta S)$, enthalpy of activation $(\Delta H)$ and free energy of activation $(\Delta G)$ are calculated and the values are given in Tables 3-5. Arrhenius plots in Figs. 3 and 4 were drawn by least square method and corresponding cor-

Table 4

Pre-exponential factors and rate constant values for thermal degradation of Co(II)-MCP complexes 
Table 5

Entropy $\Delta S$, free energy $\Delta G$ and enthalpy $\Delta H$ values for Co-MCP complexes

\begin{tabular}{|c|c|c|c|c|c|c|c|c|c|c|}
\hline \multirow[t]{2}{*}{ Compounds } & \multirow[t]{2}{*}{ Process } & \multicolumn{3}{|c|}{$\Delta S\left(\mathrm{~kJ} \mathrm{~mol}^{-} 1\right)$} & \multicolumn{3}{|c|}{$\Delta G\left(\mathrm{~kJ} \mathrm{~mol}^{-1} 1\right)$} & \multicolumn{3}{|c|}{$\Delta H\left(\mathrm{~kJ} \mathrm{~mol}^{-1}\right)$} \\
\hline & & $\mathrm{H}-\mathrm{M}$ & $\mathrm{C}-\mathrm{R}$ & Broido's & H-M & C-R & Broido’s & H-M & C-R & Broido's \\
\hline MCP & $\begin{array}{l}\text { I } \\
\text { II }\end{array}$ & $\begin{array}{r}-92.3 \\
-236.9\end{array}$ & $\begin{array}{l}-117.6 \\
-248.4\end{array}$ & $\begin{array}{r}-92.2 \\
-233.6\end{array}$ & $\begin{array}{l}134.6 \\
229.4\end{array}$ & $\begin{array}{l}139.5 \\
227.1\end{array}$ & $\begin{array}{l}134.6 \\
228.8\end{array}$ & $\begin{array}{l}83.8 \\
24.0\end{array}$ & $\begin{array}{l}74.7 \\
11.9\end{array}$ & $\begin{array}{l}83.9 \\
26.3\end{array}$ \\
\hline$\left[\mathrm{Co}(\mathrm{MCP})\left(\mathrm{H}_{2} \mathrm{O}\right)_{2}\left(\mathrm{ClO}_{4}\right)_{2}\right]$ & $\begin{array}{l}\text { I } \\
\text { II }\end{array}$ & $\begin{array}{r}59.0 \\
-218.7\end{array}$ & $\begin{array}{r}39.3 \\
-232.4\end{array}$ & $\begin{array}{r}62.4 \\
-218.9\end{array}$ & $\begin{array}{l}131.7 \\
201.0\end{array}$ & $\begin{array}{l}135.2 \\
198.6\end{array}$ & $\begin{array}{l}131.7 \\
201.0\end{array}$ & $\begin{array}{r}164.2 \\
31.8\end{array}$ & $\begin{array}{r}156.9 \\
19.0\end{array}$ & $\begin{array}{r}166.0 \\
31.8\end{array}$ \\
\hline$\left[\mathrm{Co}(\mathrm{MCP})\left(\mathrm{H}_{2} \mathrm{O}\right)_{2} \mathrm{Br}_{2}\right]$ & $\begin{array}{l}\text { I } \\
\text { II } \\
\text { III }\end{array}$ & $\begin{array}{l}-137.4 \\
-186.4 \\
-207.1\end{array}$ & $\begin{array}{l}-187.8 \\
-214.0 \\
-222.5\end{array}$ & $\begin{array}{l}-137.6 \\
-186.7 \\
-207.3\end{array}$ & $\begin{array}{r}97.4 \\
150.3 \\
259.3\end{array}$ & $\begin{array}{l}116.4 \\
156.7 \\
257.9\end{array}$ & $\begin{array}{r}97.4 \\
150.3 \\
259.3\end{array}$ & $\begin{array}{l}42.2 \\
39.4 \\
54.41\end{array}$ & $\begin{array}{l}40.9 \\
29.2 \\
37.8\end{array}$ & $\begin{array}{l}42.1 \\
39.1 \\
54.2\end{array}$ \\
\hline$\left[\mathrm{Co}(\mathrm{MCP})\left(\mathrm{H}_{2} \mathrm{O}\right)_{2} \mathrm{Cl}_{2}\right]$ & $\begin{array}{l}\text { I } \\
\text { II } \\
\text { III }\end{array}$ & $\begin{array}{l}-155.4 \\
-162.7 \\
-241.8\end{array}$ & $\begin{array}{l}-191.5 \\
-190.8 \\
-244.7\end{array}$ & $\begin{array}{l}-147.5 \\
-163.0 \\
-247.4\end{array}$ & $\begin{array}{r}94.6 \\
143.9 \\
265.6\end{array}$ & $\begin{array}{l}104.9 \\
150.3 \\
258.6\end{array}$ & $\begin{array}{r}94.4 \\
143.9 \\
266.7\end{array}$ & $\begin{array}{l}34.2 \\
50.1 \\
25.4\end{array}$ & $\begin{array}{l}30.3 \\
40.4 \\
15.5\end{array}$ & $\begin{array}{l}37.0 \\
49.9 \\
21.0\end{array}$ \\
\hline$\left[\mathrm{Co}(\mathrm{MCP})\left(\mathrm{H}_{2} \mathrm{O}\right)_{2} \mathrm{SO}_{4}\right]$ & $\begin{array}{l}\text { I } \\
\text { II } \\
\text { III }\end{array}$ & $\begin{array}{l}-122.9 \\
-183.2 \\
-252.4\end{array}$ & $\begin{array}{l}-164.0 \\
-209.0 \\
-252.7\end{array}$ & $\begin{array}{l}-122.8 \\
-182.8 \\
-252.3\end{array}$ & $\begin{array}{r}98.7 \\
144.9 \\
251.3\end{array}$ & $\begin{array}{l}108.87 \\
150.4 \\
246.1\end{array}$ & $\begin{array}{r}98.7 \\
144.9 \\
251.2\end{array}$ & $\begin{array}{l}48.5 \\
39.4 \\
15.5\end{array}$ & $\begin{array}{r}41.8 \\
30.0 \\
9.9\end{array}$ & $\begin{array}{l}48.5 \\
39.6 \\
15.5\end{array}$ \\
\hline$\left[\mathrm{Co}(\mathrm{MCP})(\mathrm{NCS})_{2}\right]$ & $\begin{array}{l}\text { I } \\
\text { II } \\
\text { III }\end{array}$ & $\begin{array}{l}-110.2 \\
-133.6 \\
-218.8\end{array}$ & $\begin{array}{l}-159.7 \\
-185.7 \\
-224.6\end{array}$ & $\begin{array}{l}-106.7 \\
-152.3 \\
-220.2\end{array}$ & $\begin{array}{l}124.5 \\
153.6 \\
266.8\end{array}$ & $\begin{array}{l}145.8 \\
183.1 \\
266.63\end{array}$ & $\begin{array}{l}124.4 \\
154.4 \\
267.0\end{array}$ & $\begin{array}{l}68.3 \\
71.11 \\
45.6\end{array}$ & $\begin{array}{l}64.4 \\
68.5 \\
39.6\end{array}$ & $\begin{array}{l}70.1 \\
60.3 \\
44.4\end{array}$ \\
\hline$\left[\mathrm{Co}(\mathrm{MCP})\left(\mathrm{H}_{2} \mathrm{O}\right)_{2}\left(\mathrm{NO}_{3}\right)_{2}\right]$ & $\begin{array}{l}\text { I } \\
\text { II } \\
\text { III }\end{array}$ & $\begin{array}{l}-161.8 \\
-180.3 \\
-220.9\end{array}$ & $\begin{array}{l}-198.9 \\
-199.5 \\
-226.3\end{array}$ & $\begin{array}{l}-159.2 \\
-161.2 \\
-220.5\end{array}$ & $\begin{array}{r}99.4 \\
153.3 \\
264.3\end{array}$ & $\begin{array}{r}99.3 \\
153.8 \\
267.6\end{array}$ & $\begin{array}{r}93.3 \\
152.3 \\
264.3\end{array}$ & $\begin{array}{l}33.7 \\
43.7 \\
43.2\end{array}$ & $\begin{array}{l}27.8 \\
42.4 \\
41.1\end{array}$ & $\begin{array}{l}33.3 \\
54.3 \\
43.5\end{array}$ \\
\hline$\left[\mathrm{Co}(\mathrm{MCP})\left(\mathrm{H}_{2} \mathrm{O}\right)_{2}\left(\mathrm{CH}_{3} \mathrm{COO}\right)_{2}\right]$ & $\begin{array}{l}\text { I } \\
\text { II } \\
\text { III }\end{array}$ & $\begin{array}{l}-133.4 \\
-128.8 \\
-190.9\end{array}$ & $\begin{array}{l}-164.2 \\
-169.8 \\
-208.5\end{array}$ & $\begin{array}{l}-136.4 \\
-127.9 \\
-192.0\end{array}$ & $\begin{array}{l}105.9 \\
148.7 \\
234.9\end{array}$ & $\begin{array}{l}120.21 \\
170.8 \\
246.1\end{array}$ & $\begin{array}{l}106.0 \\
148.7 \\
235.1\end{array}$ & $\begin{array}{l}47.8 \\
71.5 \\
61.8\end{array}$ & $\begin{array}{l}48.7 \\
68.9 \\
56.9\end{array}$ & $\begin{array}{l}46.6 \\
71.9 \\
60.9\end{array}$ \\
\hline
\end{tabular}

relation co-efficient were also calculated and tabulated in Table 3.

The activation energy values for $\mathrm{Co}(\mathrm{II})-\mathrm{MCP}$ complexes are above $35.6 \mathrm{~kJ} \mathrm{~mol}^{-1}$ for the dehydration reactions in all the three methods. These values are more than the activation energy values for the removal of lattice water [22]. The activation energy values for the second stage decomposition are in the range $38-76 \mathrm{~kJ} \mathrm{~mol}^{-1}$ for Horowitz-Metzger method. The $E_{\mathrm{a}}$ values for other two methods are also in the same range. $E_{\mathrm{a}}$ of the third step degradation also follow the same trend in all the methods (Table 3).

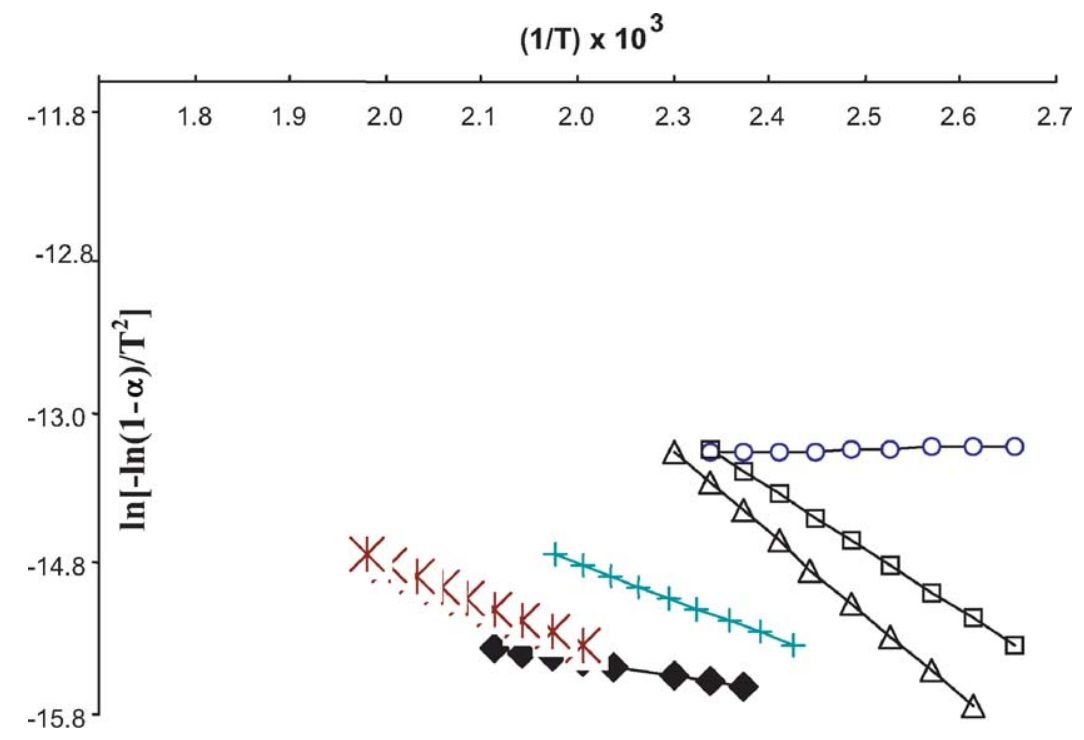

Fig. 3. Typical Coats-Redfern plot for the determination of activation energies for second step degradation of Co(II)-MCP complexes: $\left[\mathrm{Co}(\mathrm{MCP})\left(\mathrm{H}_{2} \mathrm{O}\right)_{2} \mathrm{Cl}_{2}\right](\mathrm{O}),\left[\mathrm{Co}(\mathrm{MCP})\left(\mathrm{H}_{2} \mathrm{O}\right)_{2}(\mathrm{NCS})_{2}\right](\square),\left[\mathrm{Co}(\mathrm{MCP})\left(\mathrm{H}_{2} \mathrm{O}\right)_{2}\left(\mathrm{SO}_{4}\right)\right](\triangle),\left[\mathrm{Co}(\mathrm{MCP})\left(\mathrm{H}_{2} \mathrm{O}\right)_{2} \mathrm{Br}_{2}\right](\bullet),\left[\mathrm{Co}(\mathrm{MCP})\left(\mathrm{H}_{2} \mathrm{O}\right)_{2}(\mathrm{NO})_{3}\right](+)$, $\left[\mathrm{Co}(\mathrm{MCP})\left(\mathrm{H}_{2} \mathrm{O}\right)_{2}\left(\mathrm{CH}_{3} \mathrm{COO}\right)_{2}\right]$ ( $\left({ }^{-}\right)$. 


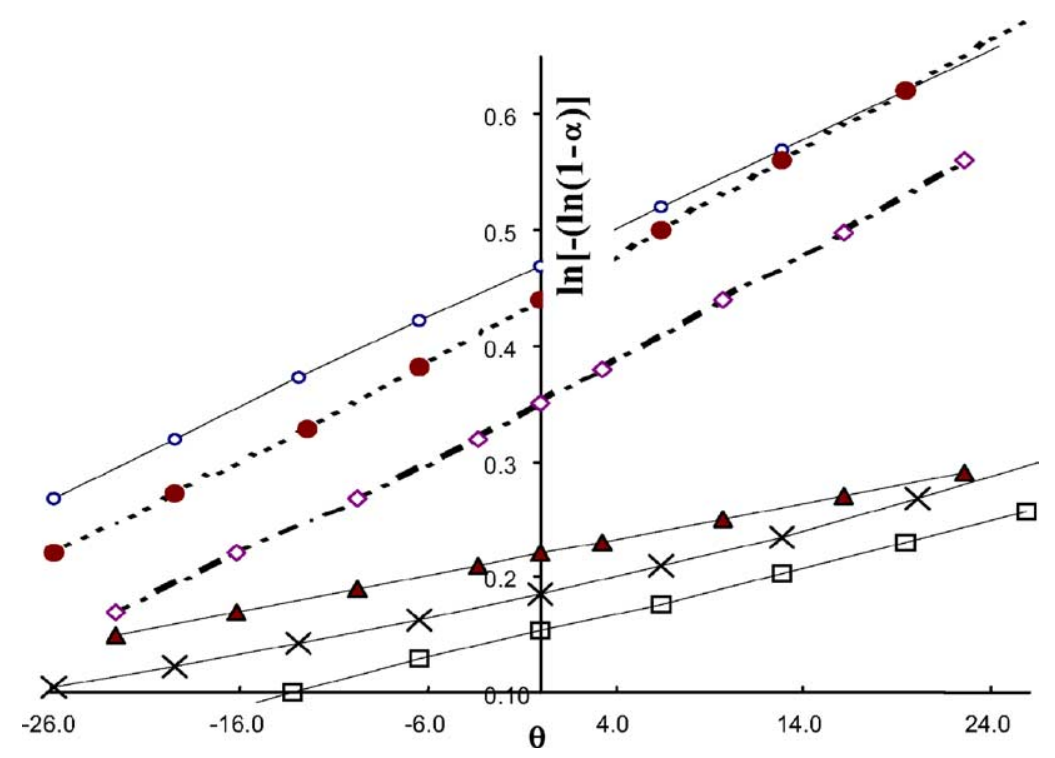

Fig. 4. Typical Horowitz-Metzger plot for the determination of activation energies for third degradation step degradation of Co-MCP complexes: $\left[\mathrm{Co}(\mathrm{MCP})\left(\mathrm{H}_{2} \mathrm{O}\right)_{2} \mathrm{Br}_{2}\right](\mathrm{O}),\left[\mathrm{Co}(\mathrm{MCP})\left(\mathrm{H}_{2} \mathrm{O}\right)_{2} \mathrm{Cl}_{2}\right](\square),\left[\mathrm{Co}(\mathrm{MCP})\left(\mathrm{H}_{2} \mathrm{O}\right)_{2} \mathrm{SO}_{4}\right](\boldsymbol{\Delta}),\left[\mathrm{Co}(\mathrm{MCP})\left(\mathrm{H}_{2} \mathrm{O}\right)_{2}\left(\mathrm{NCS}_{2}\right](\diamond),\left[\mathrm{Co}(\mathrm{MCP})\left(\mathrm{H}_{2} \mathrm{O}\right)_{2}\left(\mathrm{CH} \mathrm{COO}_{3} \mathrm{COO}(\boldsymbol{\bullet})\right.\right.\right.$, $\left[\mathrm{Co}(\mathrm{MCP})\left(\mathrm{H}_{2} \mathrm{O}\right)_{2}\left(\mathrm{NO}_{3}\right)_{2}\right](\times)$.

From the TGA thermograms it is observed that the curves for the metal chelates are broader while the curve for the ligand is steeper. On the basis of this it is assumed that the rate of the pyrolysis of metal chelate is slower than that of the ligand [23]. This fact is also supported by the rate constant values given in Table 4. The frequency factor for the removal of two coordinated water molecules is larger as they are loosely bound and can be easily removed. The pre-exponential value decreases from second to third stage degradation. The values of pre-exponential factor $\ln A$, also indicate that the decomposition of $\mathrm{Co}(\mathrm{II})-\mathrm{MCP}$ complexes were slow [24] process.

The relative sizes of inorganic ligands and the free volumes may explain the entropy effects, which are shown by the data given in Table 5. The water molecules may be able to slip into an interstitial position causing little or no lattice distortion, when there is a large difference between the size of the cation and anion. Therefore, the entropy of activation is small. When the cation and anion are of approximately the same size, water can occupy an interstitial position only with considerable lattice expansion so that the entropy of activation is more. The entropy of activation for $\mathrm{Co}$ (II)-MCP complexes follows the same order of thermal stability as that of the ligands. The lower entropy values were observed in the second stage decomposition, when compared to the values of, that of the first stage decomposition. In the second stage, it is the major portion of the MCP ligand, which is undergoing oxidation. Due to its larger size, compared to that of the water molecules there will be larger distortion in the lattice structure of the remaining portion of the molecule. Hence, second step degradation has lower entropy values than that of the first step degradation. Once again the increase in the entropy values in the third step degradation may be due to the smaller sizes of inorganic ligands and remaining portions of the MCP ligand.

\section{Conclusion}

A new series of $\mathrm{Co}$ (II) complexes were prepared and characterized by the elemental analysis and spectral studies, based on these studies they have been assigned octahedral geometry. TGA data supports the presence of coordinated water. The thermal stability of the complexes was explained considering the transition temperature and IPDT values. For cobalt(II)-MCP complexes, the stability decreases in the order perchlorate $>$ acetate $>$ sulphate $>$ bromide $>$ chloride $>$ nitrate. The decomposition of all the complexes takes place in three steps, except perchlorate complex. The effect of anions in the deaquation reaction can be explained by defect diffusion model. On the basis of this model the stability towards deaquation reaction decreases in the order $\mathrm{ClO}_{4}{ }^{-}>\mathrm{CH}_{3} \mathrm{COO}^{-}>\mathrm{SCN}^{-}>\mathrm{SO}_{4}{ }^{2-}>\mathrm{Br}^{-}>\mathrm{Cl}^{-}>$ $\mathrm{NO}_{3}{ }^{-}$.

\section{Acknowledgements}

The authors express their thanks to Astra IDL Ltd., Bangalore for gift sample of metoclopramide, Dr. S.T. Aruna and Dr. Prasanna for IR spectra and elemental analysis.

\section{References}

[1] M. Murakami, N. Inukai, A. Koda, K. Nakano, Chem. Pharm. Bull. 19 (8) (1971) 1696. 
[2] L. Anker, J. Lauterwein, H.V. de Waterbeemed, B. Bernard Testa, Helv. Chim. Acta 67 (1984) 706.

[3] P.G. Ramappa, K.S. Ramachandra, J. Asian Chem. 1 (1989) 19.

[4] P.G. Ramappa, K.S. Ramachandra, J. Inst. Chem. 59 (1987) 115.

[5] H. Horowitz, G. Metzger, Anal. Chem. 35 (1964) 68.

[6] A.W. Coats, J.P. Redfern, Nature 201 (1964) 68.

[7] A. Broido, J. Poly. Sci. A 2 (1969) 1761.

[8] A.I. Vogel, A Text Book of Quantitative Inorganic Analysis, 4th ed., Longmans Green, ELBS, London, p. 462.

[9] M.M. Kotan, Y.N. Sazonov, Polym. Sci. USSR 15 (1973) 1857.

[10] N. Calu, L. Odochain, G.L. Brinzan, N. Bilba, J. Them. Anal. 30 (1985) 547.

[11] H.S.B. Naik, Siddaramaiah, P.G. Ramappa, J. Therm. Anal. Calorimetry 55 (1998) 841-849.

[12] M. Nath, R. Yadav, Synth. React. Met. Org. Chem. 25 (9) (1995) 1529.

[13] J.E. House Jr., Thermochim. Acta 38 (1981) 59.

[14] A.R. West, Solid State Chemistry and its Applications, Wiley, New York, 1984, pp. 318-333.
[15] H. Schmalzried, Solid State Reactions, 2nd ed., VEB Chemie, Weinheim, 1974, pp. 24-27.

[16] J. Casabo, T. Flor, F. Teixidor, J. Ribas, Inorg. Chem. 25 (1986) 3166.

[17] M. Mori, R.T. Tsuchiya, Bull. Chem. Soc. Jpn. 33 (1960) 841.

[18] P.G. Ramappa, K.G. Somasekharappa, Synth. React. Inorg. Met. Org. Chem. 22 (1992) 1267.

[19] H.S. Bhojya Naik, Siddaramaiah, P.G. Ramappa, Thermochim. Acta 2998 (1996) 1.

[20] C. Pariya, A. Gosh, N.R. Chaudhuri, Thermochim. Acta 273 (1996) 185.

[21] A. Ohyoshi, S. Hiraki, T. Odate, S. Kohata, J. Oda, Bull. Chem. Soc. Jpn. 48 (1) (1975) 262.

[22] K.K. Mohammed Yusuff, R. Sreekala, Thermochim. Acta 179 (1991) 313.

[23] K.H. Halawani, K.M. Abd El-Salaam, F.A. Fakiha, Int. J. Chem. 4 (1992) 201

[24] R.K. Bansal, R. Agrawal, K. Keshav, Angew. Makromol. Chem. 117 (1983) 211 